powered trial to detect any meaningful superiority of spinal cord stimulation for RA patients.

\section{THE ASSOCIATION OF NIGHT-TIME SYSTOLIC BLOOD PRESSURE WITH ULTRASOUND MARKERS OF SUBCLINICAL CARDIAC AND VASCULAR DAMAGE}

${ }^{1}$ Anne Marie O'Flynn*, ${ }^{2}$ Ronan Curtin, ${ }^{1}$ Patricia Kearney. ${ }^{1}$ University College Cork; ${ }^{2}$ Cork University Hospital; *Presenting Author

\subsection{6/heartjnl-2016-309890.92}

Background Ambulatory blood pressure monitoring (ABPM) measures blood pressure (BP) over a prolonged period and has been shown to be superior to office BP for the prediction of clinical events. In particular, night-time systolic BP is a stronger predictor than systolic daytime BP. It is not yet clear if night-time BP should be a specific therapeutic target although some studies have demonstrated promising results. Subclinical cardiovascular disease is a prognostic marker for future cardiovascular events. Our aim is to examine the association of night-time systolic BP with subclinical cardiac dysfunction measured by global longitudinal strain (GLS) and subclinical vascular damage measured by carotid intima media thickness (CIMT) and carotid plaques.

Methods In 2014 a random sample of 80 individuals, stratified by BP status at baseline recruitment to the Mitchelstown Cohort Study, were invited to undergo repeat ABPM, echocardiogram and carotid ultrasound. ABPM was performed using the Spacelabs 90217 monitor. GLS was measured by speckletracking analysis of echocardiogram images carried out on a Philips iE33 ultrasound machine. Mean CIMT was measured at the distal $1 \mathrm{~cm}$ of the common carotid artery. Still images were taken from 3 angles on both sides of the neck using a Philips Cx50 ultrasound machine. The presence of carotid plaques was recorded. Philips QLAB cardiac and vascular ultrasound quantification software was used for analysis. The association of night-time systolic BP with GLS, CIMT and carotid plaques was assessed using linear and logistic regression.

Results Fifty (response rate 63\%) individuals took part in this study. In univariable models night-time systolic BP was significantly associated with GLS (Beta coefficient 0.85 for every $10 \mathrm{mmHg}$ rise, 95\% CI 0.3-1.4) and carotid plaques (OR 1.9 for every $10 \mathrm{mmHg}$ rise, 95\% CI 1.1-3.2). Univariable analysis of daytime systolic BP did not demonstrate any statistically significant associations. In age and sex adjusted models, the association for night-time systolic BP and GLS remained significant (Beta coefficient 0.7 for every $10 \mathrm{mmHg}$ rise, 95\% CI $0.1-1.3)$. The association for carotid plaques was no longer statistically significant. In multivariable models findings were diminished.

Discussion Our results support an association between nighttime systolic BP and subclinical cardiac and vascular disease. However this is a small study which limits generalisability and the sample size may have provided insufficient power to detect true associations between night-time systolic BP and target organ damage in multivariable models and CIMT in particular. The use of ABPM and ultrasound technology may help guide therapeutic decisions in those with hypertension. When assessing ABPM results the absolute night-time BP seems to be the most important parameter but ultimately a large randomised controlled trial involving chronotherapy is necessary to fully address this.

\section{3 A DOUBLE-BLIND PLACEBO-CONTROLLED RANDOMISED STUDY OF THE EFFECTS OF CANDESARTAN VERSUS AMLODIPINE ON CAPILLARY RAREFACTION IN ESSENTIAL HYPERTENSION}

${ }^{1}$ Rajendra Raghuraman, ${ }^{2}$ Christine Carney, ${ }^{2}$ Oluwabusola Ogunseitan, ${ }^{2}$ Helen Mullahy, ${ }^{3}$ Duolao Wang, ${ }^{4}$ Tarek Antonios*. 'St. George's, University of London; ${ }^{2}$ St. George's University Hospitals NHS Foundation Trust; ${ }^{3}$ Liverpool School of Tropical Medicine; ${ }^{4}$ SGUL; *Presenting Author

\subsection{6/heartjnl-2016-309890.93}

Background A reduction in the density of capillaries (rarefaction) is known to occur in many tissues in patients with essential hypertension (HTN) and plays a crucial role in increasing peripheral resistance and blood pressure (BP). The aim of this clinical trial was to assess in a controlled, double blind, placebo-controlled design the effects of treatment of HTN with candesartan or amlodipine on microvascular rarefaction and other indices of vascular function in individuals with mild-to-moderate essential HTN.

Methods The capillary microcirculation was studied using the well-validated intravascular microscopy technique. After a 2week single-blind placebo run-in period, patients who remained hypertensive (systolic BP 140-180 $\mathrm{mmHg}$ and/or diastolic BP $90-110 \mathrm{mmHg}$ ) were randomised to 8 -weeks treatment with either candesartan $8 \mathrm{mg}$ daily (with forced titration to $16 \mathrm{mg}$ after 2 weeks) or amlodipine $5 \mathrm{mg}$ orally daily (with forced titration to $10 \mathrm{mg}$ after 2 weeks). Other vascular measurements included pulse wave velocity with Complior machine, central BP and Aortic Augmentation Index measurements with Omron HEM-9000AI machine.

Results Treatment with candesartan and amlodipine significantly reduced both brachial and central BP at 4 and 8 weeks (mean change $-19.0 \mathrm{mmHg}$; 95\% CI -11.1 to --26.9 , p < 0.0001 ), and to 8 weeks active treatment (mean change -26.3 $\mathrm{mmHg} ; 95 \% \mathrm{CI}-17.5$ to $-35.0, \mathrm{p}<0.0001$ ) but had no significant effect on basal (functional) or maximal (structural) capillary densities. Both drugs also reduced central BP and Aortic augmentation index significantly after 4 and 8 weeks but there was no significant changes in PWV.

Conclusions The study confirms that 8 weeks treatment with either candesartan or amlodipine significantly reduces radial and central BP in essential HTN but may not be a sufficient circumstance for inducing a regression in microvascular abnormalities.

\section{FLOW-CONTRACTION MATCHING IN THE HUMAN HEART: A NOVEL INVASIVE STUDY OF THE COMPLEX CARDIAC-CORONARY INTERACTION IN ISCHAEMIC HEART DISEASE}

${ }^{1}$ Tiffany Patterson*, ${ }^{2}$ Simone Rivolo, ${ }^{2}$ Satpal Arri, ${ }^{3}$ Brian Clapp, ${ }^{1}$ Divaka Perera ${ }^{2}$ Michael Marber, ${ }^{2}$ Jack Lee, ${ }^{3}$ Simon Redwood. ${ }^{1}$ KCL/St Thomas' Hospital; ${ }^{2}$ King's College London; ${ }^{3}$ St Thomas' Hospital; *Presenting Author

\subsection{6/heartjnl-2016-309890.94}

Background A first-in-human study of simultaneous invasive real-time left ventricular (LV) and coronary haemodynamics in the cardiac catheter lab enabling accurate delineation of the complex cardiac-coronary interaction and influence of antianginal therapy in coronary artery disease (CAD).

Method 15 patients completed the protocol (Figure;A\&B). Coronary measurements (baseline and $1 \mathrm{mg}$ isosorbide 